\title{
On Analysis of DSC Curves for Characterization of Intrinsic Properties of NiTi Shape Memory Alloys
}

\author{
A. ZióŁKOWSKI* \\ Institute of Fundamental Technological Research, Polish Academy of Sciences \\ Pawińskiego 5B, 02-106 Warsaw, Poland
}

\begin{abstract}
Differential scanning calorimetry is discussed as a tool for characterization of shape memory alloy materials with the example of $\mathrm{Ni}_{51 \text { at.\%- }} \mathrm{Ti}$ polycrystalline material. Some inconsistencies connected with the differential scanning calorimetry analysis results present in the contemporary literature are indicated, for example large discrepancies in the registered heats of phase transition or differences in registered heats of forward and reverse phase transition, especially in the case of multi-stage phase transition sequences. An attempt is undertaken to explain some of the discrepancies. The overall conclusion from the present work is that forward and reverse sensible heats of phase transition are equal, and discrepancies reported in the literature are artefacts. It is recommended that as a standard practice of differential scanning calorimetry analysis of shape memory alloy materials not only characteristic temperatures but also sensible heat of phase transition should be reported, which can be done at practically no additional cost.
\end{abstract}

PACS: 05.70.Ln, 81.30.Kf, 81.70.Pg, 82.60.Fa, 62.20.fg, 87.85.J-

\section{Introduction}

Shape memory alloy (SMA) materials arouse the interest of researchers and engineers due to a very complex behaviour: interesting and useful properties exhibiting themselves in the form of pseudoelasticity (PE) or one-way shape memory effect (SME). The most popular among many SMA materials are NiTi based alloys, which besides excellent and stable PE and SME possess valuable features such as corrosion resistance, high strength, stability of response, and biocompatibility. The special features of SMA materials stem from their crystallographically reversible thermoelastic martensitic transformation and in the specific case of NiTi based alloys phase transformation of austenite $(B 2)$ into martensite $\left(B 19^{\prime}\right)$ through a single-stage phase transition $(\mathrm{PT})(\mathrm{A} \rightarrow \mathrm{M})$ or a multi-stage PT, austenite $\rightarrow$ R-martensite $\rightarrow$ monoclinic martensite $(\mathrm{A} \rightarrow \mathrm{R} \rightarrow \mathrm{M})$. The $\mathrm{PT}$ may be induced by either stress or temperature. Thus the PE and SME phenomena involve strong coupling between different physical fields. Some researchers distinguish even more multi-stage PTs each of martensitic character [1, 2]. The functional and physical properties of SMA materials such as SME, PE, wear resistance, functional fatigue, size of hysteresis loop, and level of recovery stress/strain are very strongly influenced by thermomechanical treatment (TMT). Nearly all techniques known in materials science, such as work hardening, annealing and ageing, and introduction of chemically and physically inert inclusions, find application here. The NiTi materials are extremely sensitive to small variations in chemical composition and/or "impurities" content (O, C, H) [3, 4]. A kind of byproduct of functional features optimization

* e-mail: aziolk@ippt.gov.pl achieved through TMT is the appearance of single- or multi-stage PT, change of microstructure, and change in the characteristic temperatures of PT. These last parameters practically decide whether a particular SMA material will qualify for specific medical or non-medical application $[5,6]$. Proper characterization of SMA materials is quite difficult as a commonly accepted theoretical model that comprehensively covers various aspects of their behavior is at present non-existent. However, models covering a partial range of SMA behavior can facilitate this task, for example, the thermodynamic $R_{L}$ model of SMA pseudoelastic behavior [7].

In the present work the $R_{L}$ model is used to link characteristic temperatures determined from differential scanning calorimetry (DSC) testing of the austenite start and the martensite start at null stress, $A_{s}^{0}$ and $M_{s}^{0}$, and the sensible heat of PT $\Delta u^{*}$ with modeling parameters characterizing useful properties of SMA material, for example, internal dissipation. This is elucidated using the results of DSC tests run on $\mathrm{Ni}_{51 \text { at.\% }}$-Ti SMA wire submitted to cold work followed by various ageing treatments. An attempt is made to explain the considerable difference in sensible heats of forward and reverse PTs reaching $20 \%$ or more encountered in the literature devoted to DSC characterization of SMA materials.

\section{Experimental}

The DSC tests were performed on a commercial, polycrystalline NiTi alloy wire provided by @mt Company (Belgium) with a diameter of $1.0 \mathrm{~mm}$ and nominal composition of 51.0 at.\% Ni. Pieces of as-received wire $(30 \%$ last stage cold-worked) several centimetres long were aged in an argon atmosphere for $15 \mathrm{~min}$ and $30 \mathrm{~min}$ at temperatures of 400,450 , and $500{ }^{\circ} \mathrm{C}$, respectively. The transformation temperatures and sensible heats of 
the as-received and aged specimens were determined by DSC (TA 2920 CE apparatus). DSC samples with masses close to $30 \mathrm{mg}$ were taken from the heat-treated materials. They were heated to $120^{\circ} \mathrm{C}$, cooled to $-120^{\circ} \mathrm{C}$, and heated to $120^{\circ} \mathrm{C}$ again. The cycle was repeated twice to check the repeatability of the material response. Several heating and cooling rates were used: 5, 10, and $20^{\circ} \mathrm{C} / \mathrm{min}$, but only the results of tests performed at a rate of $10^{\circ} \mathrm{C} / \mathrm{min}$ are reported here. Using TA Universal Analysis software, the phase transformation temperatures were determined in a standard way with the aid of tangents, and sensible heats of PT were determined as respective areas under DSC curves.

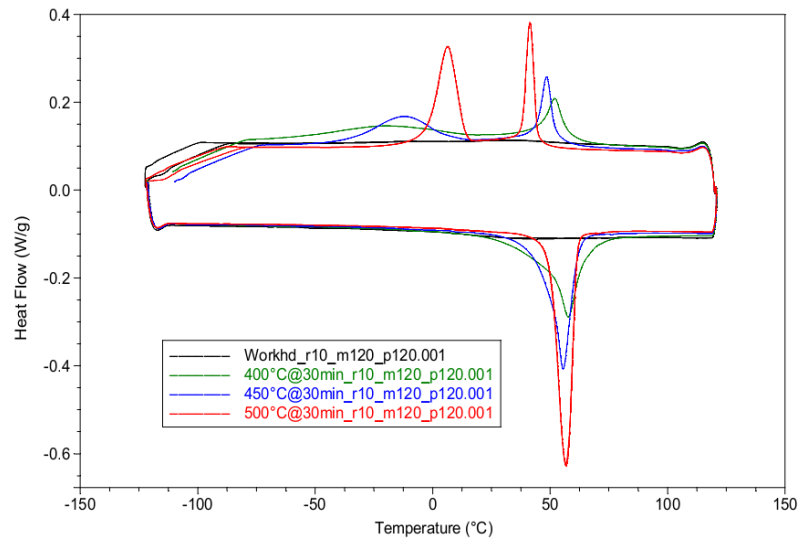

Fig. 1. Comparison of DSC curves obtained for Ni51Ti wire $30 \%$ cold-worked and submitted to ageing heat treatments for $30 \mathrm{~min}$ at 400,450 , and $500{ }^{\circ} \mathrm{C}$.

Overlaid DSC charts for specimens made of as-received materials ( $30 \%$ cold-worked) and aged for $30 \mathrm{~min}$ at 400 , 450 , and $500^{\circ} \mathrm{C}$ are shown in Fig. 1. It can be noticed that cold-worked material is SME "dead"; that is, it exhibits no PT. Increasing the ageing temperature at a constant processing time of $30 \mathrm{~min}$ results in more and more pronounced two-stage forward and one-stage reverse PT with increasing sensible heats of $22.5,23.6$, and $27.0 \mathrm{~J} / \mathrm{g}$, respectively. It is interesting to note that Ni51at.\%-Ti alloy, not from the same batch, which was solutionized $\left(1000^{\circ} \mathrm{C}, 30 \mathrm{~min}\right)$ exhibited both forward and reverse single-stage transformation with an unexpectedly small heat of transformation $5.5 \mathrm{~J} / \mathrm{g}$, which was four to five times smaller than in the case of the aged specimen.

\section{Results and discussion}

Sensible heats of PT of NiTi materials (50.6$51.2 \mathrm{Ni}$ at.\%) optimized with respect to their SMA properties typically reported in the literature are at the level of $20 \mathrm{~J} / \mathrm{g}$. However, quite frequently the heats of forward and reverse transition differ by over $20 \%$ (see e.g. [8]). In order to evaluate the credibility of this information the present author performed a literature survey and undertook his own analyses. These convinced him that two conjectures can be credibly accepted for NiTi alloys that are DSC tested in a temperature range below approximately $200^{\circ} \mathrm{C}$.

Hypothesis H1: The specific internal energy of the NiTi sample before the DSC test at state 1 is the same as internal energy $u_{3}$ at termination of the DSC test cycle at state 3 ; that is, $u_{1}(\varepsilon, s, z)=u_{3}(\varepsilon, s, z) \Leftrightarrow \Delta u=0$ (see Fig. 2).

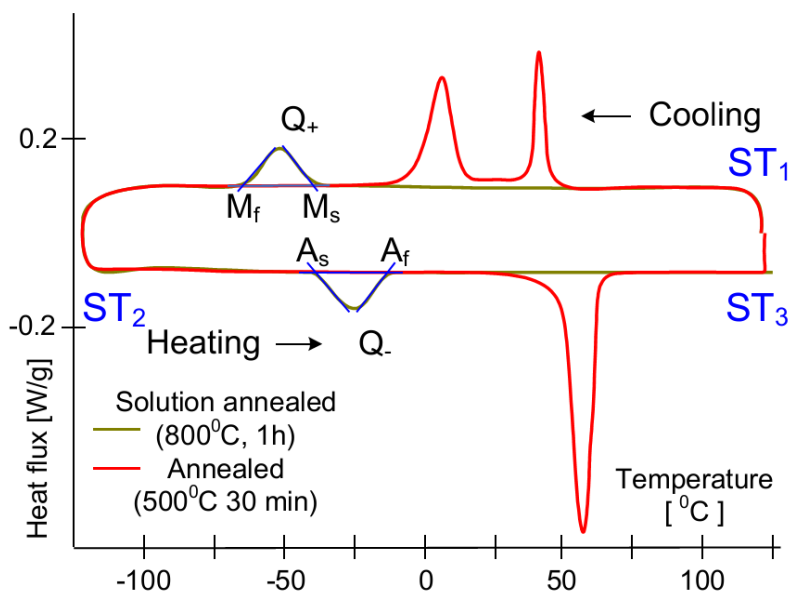

Fig. 2. Schematic view of DSC tests results for NiTi alloy submitted to different TMTs. In solutionized state, single-stage forward and reverse $\mathrm{PT}$ takes place, but the specimen aged for $30 \mathrm{~min}$ at $500{ }^{\circ} \mathrm{C}$ exhibits two-stage forward and one-stage reverse PT.

Hypothesis H2: It is accepted that no net macroscopic work is done as a result of the DSC test cycle; that is, $\Delta W=\oint(\sigma / \rho) \mathrm{d} \varepsilon=0$. This conjecture is justified by well known information that so-called self-accommodating martensite induced during the DSC test exhibits null transformation strain in the presence of the very low stress remaining in the case of the DSC procedure at the level of atmospheric pressure. Considering conjectures $H 1$ and $H 2$ and the first law of thermodynamics,

$$
\begin{gathered}
\oint \mathrm{d} u=\oint \mathrm{d} q+\oint(\sigma / \rho) \mathrm{d} \varepsilon \Leftrightarrow \Delta u=\Delta Q+\Delta W, \\
\Delta Q=\Delta Q_{+}+\Delta Q_{-}=0 \Leftrightarrow \Delta Q_{+}=-\Delta Q_{-} ; \\
\Delta Q_{+}=\int_{T 1>T 2}^{T 2} \mathrm{~d} q, \quad \Delta Q_{-}=\int_{T 2}^{T 1} \mathrm{~d} q .
\end{gathered}
$$

Hence, the sensible heat of forward PT $\Delta Q_{+}$registered by DSC during the cooling stage of the test from temperature $T_{1}>M_{s}^{0}$ to temperature $T_{2}<M_{f}^{0}$, which is removed to the surroundings, must be equal in value to the sensible heat of reverse $\mathrm{PT} \Delta Q_{-}$registered by DSC during the heating stage of the test from temperature $T_{2}<A_{s}^{0}$ to temperature $A_{f}^{0}<T_{1}$, which is absorbed from the surroundings.

Let us analyze the DSC test result obtained for the $\mathrm{NiTi}$ alloy heat-treated for $30 \mathrm{~min}$ at $500^{\circ} \mathrm{C}$ and water quenched. When the forward R-phase transition and M-phase transition are treated separately, then the 
following values of sensible heats for forward $\Delta Q_{+}=$ $\Delta Q_{+}^{\mathrm{R}}+\Delta Q_{+}^{\mathrm{M}}=7.1+13.6=20.7 \mathrm{~J} / \mathrm{g}$ and reverse $\Delta Q_{-}=26.9 \mathrm{~J} / \mathrm{g}$ PT are obtained (see Fig. 3a). There is a mismatch of about $23 \%$ between the forward and reverse PT sensible heats. However, a closer look at the chart in Fig. 3 allows one to notice that the R-phase and M-phase transitions mutually interact in multi-stage transition and should rather be treated together when evaluating the total heat of transformation. When this is done in the DSC analysis the following sensible heats are obtained: $\Delta Q_{+}=26.8 \mathrm{~J} / \mathrm{g}$ and $\Delta Q_{-}=27.0 \mathrm{~J} / \mathrm{g}$. A mismatch in the level of DSC apparatus accuracy is in accordance with the conclusion drawn from the suppositions of hypotheses $H 1$ and $H 2$.

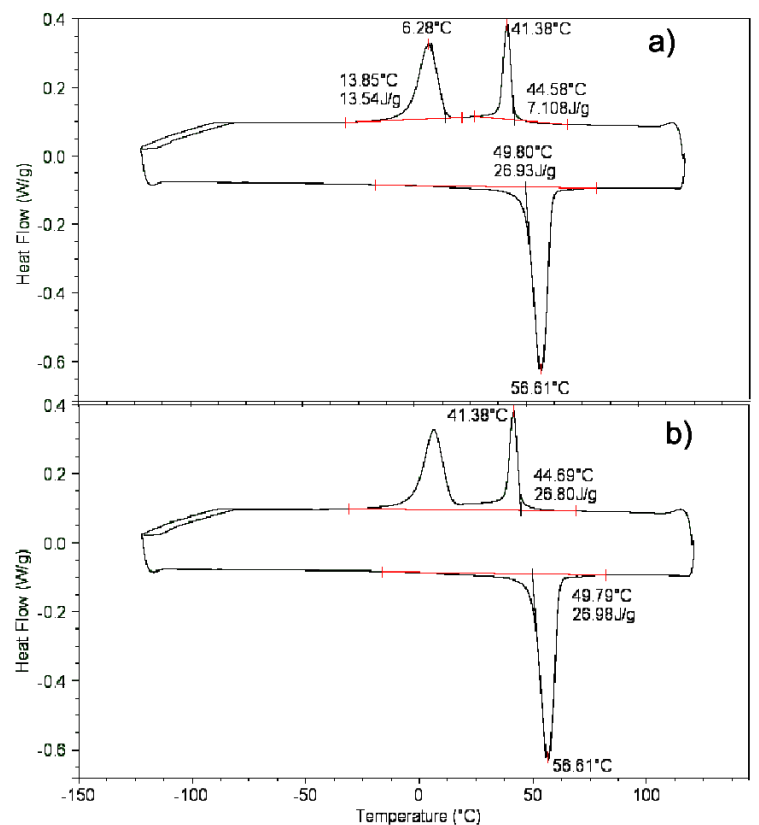

Fig. 3. DSC test results and analyses for NiTi alloy heat-treated for $30 \mathrm{~min}$ at $500{ }^{\circ} \mathrm{C}$ and water quenched. (a) R-phase and M-phase transitions are treated separately; (b) R-phase and M-phase transitions are treated as one multi-stage transition.

In order to evaluate the intrinsic properties of SMA material, use must be made of some model of SMA material behavior. The thermal equations of state of the $\mathrm{R}_{\mathrm{L}}$ thermodynamic model of pseudoelasticity obtained by formal differentiation of the Gibbs potential with respect to material state parameters are as follows (cf. [7]):

$$
\begin{aligned}
& -\partial G / \partial z \equiv \pi^{f}(\sigma, T, z) \\
& \quad=\pi_{0}^{f}(T)+\gamma \sigma_{\text {ef }} f(y) / \rho-(1-2 z) \phi_{\text {it }}(T), \\
& -\partial G / \partial T \equiv s=s_{0}^{*(1)}-z \Delta s^{*}+c_{p} \ln \left(T / T_{0}\right) \\
& \quad+\sigma \alpha / \rho+z(1-z) \bar{s}_{0}, \\
& -\partial(\rho G) / \partial \sigma \equiv \varepsilon=\alpha \Delta T+M \sigma+\varepsilon^{\mathrm{T}},
\end{aligned}
$$

where $\pi^{f}$ is the thermodynamic driving force (tdf) of phase transformation, $\sigma$ is macroscopic stress, $\pi_{0}^{f}=$ $\Delta u^{*}-T \Delta s^{*}$ is chemical Gibbs free energy, $\gamma$ is the am- plitude of pseudoelastic flow, $\sigma_{\text {ef }}$ is effective stress, $\rho$ is density, $z$ is the volume fraction of the martensitic phase, $\phi_{\text {it }}(T)=\bar{u}_{0}-T \bar{s}_{0}$ is the so-called coherent energy characterized by two constant material parameters $\bar{u}_{0}$ and $\bar{s}_{0}$, $s$ is the specific entropy of the two-phase representative volume element (RVE) of SMA material, $c_{p}$ is heat capacity, $\alpha$ is the thermal expansion coefficient, $\varepsilon$ is total macroscopic strain, $M$ is the elastic compliance tensor, and $\varepsilon^{\mathrm{T}}$ is PT strain. It can be shown that the power of heat of the SMA macro element which must be exchanged with the environment during the progress of any thermodynamic process amounts to

$$
\begin{gathered}
\dot{q}=c_{p} \dot{T}-\dot{q}_{\mathrm{tr}}, \quad \dot{q}_{\mathrm{tr}} \equiv-T \alpha \dot{\sigma} / \rho+c_{f}^{*} \dot{z}+\pi^{f} \dot{z}, \\
c_{f}^{*}(z) \equiv T\left[\Delta s^{*}-(1-2 z) \bar{s}_{0}\right],
\end{gathered}
$$

where $c_{f}^{*} \dot{z}$ describes heat power, which would be generated in a virtual, infinitesimal, thermodynamically reversible process of PT as a result of the existence of latent heat of PT. The $\dot{q}$ is positive when expelled to environment. The rate of dissipation of mechanical work $\dot{D}$ can be expressed as follows:

$$
\begin{aligned}
& \dot{D}=\pi^{f} \dot{z}=\left(c_{f}-c_{f}^{*}\right) \dot{z}, \\
& \quad c_{f}(\sigma, z) \equiv \gamma \sigma_{\text {ef }} f(y) / \rho+\Delta u^{*}-(1-2 z) \bar{u}_{0},
\end{aligned}
$$

where $c_{f} \dot{z}$ describes actual heat power, which would be generated in the real, irreversible, infinitesimal process of phase transformation resulting from the existence of the latent heat of phase transformation and dissipation of mechanical work. The total heat registered in the DSC thermograph during forward and reverse PT, respectively, neglecting thermal expansion $\alpha=0$, can be expressed by the formulae

$$
\begin{aligned}
& \Delta Q_{+}=\int_{z=0}^{z=1} c_{f} \mathrm{~d} z=\int_{z=0}^{z=1}\left[\Delta u^{*}-(1-2 z) \bar{u}_{0}\right] \mathrm{d} z \\
& \quad=\Delta u^{*}, \\
& \Delta Q_{-}=\int_{z=1}^{z=0} c_{f} \mathrm{~d} z=\int_{z=1}^{z=0}\left[\Delta u^{*}-(1-2 z) \bar{u}_{0}\right] \mathrm{d} z \\
& \quad=-\Delta u^{*} .
\end{aligned}
$$

The term $c_{p}\left(T-T_{0}\right)$ related to the heat capacity of the tested material is omitted in (5) to conform with the DSC test results, as in DSC thermographs of modern calorimeters this term is absent due to their "baseline" feature [9]. By use of $(2)_{1}$ and accepting $\bar{s}_{0}=0$, the following connections can be obtained:

$$
\begin{aligned}
& \pi^{f}(\sigma=0, z=0)=0 \Rightarrow M_{s}^{0}=\left(\Delta u^{*}-\bar{u}_{0}\right) / \Delta s^{*}, \\
& \pi^{f}(\sigma=0, z=1)=0 \Rightarrow A_{s}^{0}=\left(\Delta u^{*}+\bar{u}_{0}\right) / \Delta s^{*} .
\end{aligned}
$$

Relations (6) allow the determination of the values of the difference in the specific entropy of the austenitic and martensitic phases $\Delta s^{*}$ and the coefficient of energy of coherence $\bar{u}_{0}$ as follows:

$$
\begin{aligned}
& \Delta s^{*}=2 \Delta u^{*} /\left(A_{s}^{0}+M_{s}^{0}\right), \\
& \bar{u}_{0}=\Delta s^{*}\left(A_{s}^{0}-M_{s}^{0}\right) / 2 .
\end{aligned}
$$

Thus, it is clear that it is coherent energy $\left(\bar{u}_{0}\right)$ that is responsible for the splitting of $M_{s}^{0}$ and $A_{s}^{0}$ temperatures. Internal dissipation sources of SMA materials can be divided into thermostatic ones, quantitatively character- 
ized in the model $\mathrm{R}_{\mathrm{L}}$ by $\bar{u}_{0}$, and kinetic ones, linked with the specific route of progress of $\mathrm{PT}$ and characterized by phase transformation kinetics functions and constants. This is schematically illustrated in Fig. 4. The dissipation of mechanical work related with thermostatic sources is directly proportional to the area $A-F-D-C$ (see Fig. 4), and dissipation related with PT kinetics is directly proportional to the areas $A-B-C-A$ in the case of forward and DEFD in the case of reverse PT.

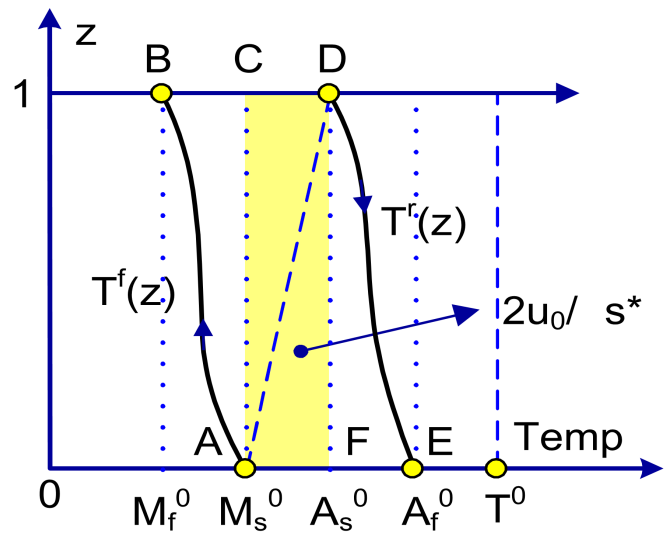

Fig. 4. Schematic view of PT during DSC test with marked areas proportional to dissipation of mechanical work.

Let us return to the determination of $\bar{u}_{0}$ and $\Delta s^{*}$ from the DSC data in the case of multi-stage - here two-stage - PT, which can create certain difficulties. In the case of two-stage PT the sensible heat determined from the DSC thermograph when treating $\mathrm{PT}$ as a whole is $\Delta u^{*}=$ $28 \mathrm{~J} / \mathrm{g}$ (see Fig. 3b). It is subsequently subdivided into two parts proportional to the heats determined when the R-phase and M-phase transitions are treated separately: $\Delta Q_{+}^{\mathrm{M}} / \Delta Q_{+}^{\mathrm{R}}=13.6 / 7.1 \approx 2\left(\Delta u^{*}=28 \Rightarrow \Delta u_{\mathrm{R}}^{*}=9000\right.$, $\Delta u_{\mathrm{M}}^{*}=18000 \mathrm{~J} / \mathrm{kg}$ ) (see Fig. 3a). On determination of the characteristic temperatures of the R-phase, M-phase, and A-phase $\left(A_{s}^{0}=323, M_{s}^{0}=287, R_{s}^{0}=318 \mathrm{~K}\right)$ and substitution of these data into formulae (7), the following are obtained:

$$
\begin{aligned}
& \Delta s^{*}=\Delta s_{\mathrm{M}}^{*}+\Delta s_{\mathrm{R}}^{*}=59+28.1=87.1 \mathrm{~J} /(\mathrm{kg} \mathrm{K}), \\
& \bar{u}_{0}=\bar{u}_{0 \mathrm{M}}+\bar{u}_{0 \mathrm{R}}=1062+70.3 \mathrm{~J} / \mathrm{kg} .
\end{aligned}
$$

In order to evaluate internal dissipation of the SMA material linked with the specific route of PT progress, information from the DSC curves is insufficient. Additional information in the form of PT kinetics relations of thermoelastic martensitic transformation is necessary. When these relations are known in dependence on the temperature, $z(T)$ and can be analytically inverted as $T(z)=z^{-1}(T)$, then sensible heats resulting from SMA cooling and heating processes, respectively, can be expressed as follows:

$$
\Delta Q_{+}=\int \mathrm{d} q=c_{p}\left(T_{0}-M_{f}^{0}\right)+\Delta u^{*}=-\Delta Q_{-} .
$$

The heats emitted and absorbed during virtual, thermo- dynamically reversible forward and reverse PT processes are

$$
\begin{aligned}
& \Delta Q_{+}^{0}=\int \mathrm{d} q^{0}=c_{p}\left(T_{0}-M_{f}^{0}\right)+\Delta s^{0} \int_{0}^{1} T^{f}(z) \mathrm{d} z, \\
& \Delta Q_{-}^{0}=-\int \mathrm{d} q^{0}=-c_{p}\left(T_{0}-M_{f}^{0}\right) \\
& -\Delta s^{0} \int_{0}^{1} T^{r}(z) \mathrm{d} z .
\end{aligned}
$$

Finally, the dissipation of mechanical work during forward and reverse PT can be estimated from the formulae

$$
\begin{gathered}
D_{+}=\Delta Q_{+}-\Delta Q_{+}^{0}=\Delta u^{*}-\Delta s^{0} \int_{0}^{1} T^{f}(z) \mathrm{d} z \geq 0, \\
D_{-}=\Delta Q_{-}-\Delta Q_{-}^{0}=\Delta s^{0} \int_{0}^{1} T^{r}(z) \mathrm{d} z-\Delta u^{*} \geq 0 .
\end{gathered}
$$

When it is justified to accept that forward and reverse PTs take place at nearly constant temperatures, that is, $T^{f}=M_{s}^{0}=M_{f}^{0}, T^{r}=A_{s}^{0}=A_{f}^{0}$, then the dissipation of mechanical work originates only from thermostatic sources and amounts to $D=2 \bar{u}_{0}$.

\section{Conclusions}

Reanalysis of experimental DSC data run on SMA specimens available in the literature and analysis of the researcher's own DSC test results run on $\mathrm{Ni}_{51}-\mathrm{Ti}$ alloy with the aid of the thermodynamic model of SMA behavior indicate that the DSC cycle experienced by NiTi materials with an upper temperature not exceeding $200^{\circ} \mathrm{C}$ can be treated as a closed thermodynamic cycle; that is, with good accuracy, the initial state of the sample material is the same as the end state after DSC test termination. For that reason the sensible heat of forward PT, regardless of whether it is single- or multi-stage, must be the same as the sensible heat of reverse single- or multi-stage PT. Thus experimentally determined values of these heats may deviate in value by a factor of 1 to $2 \%$, that is, the typical accuracy of current DSC apparatuses.

The multi-stage transitions of R-phase (one or more) and M-phase appearing in Ni rich NiTi alloys after some ageing treatments should be first treated as single transformation when determining the sensible heat of forward or reverse PT, as individual (crystallographically different) transitions strongly mutually interact. Any decoupling and attribution of sensible heats to particular PT should be made at a later stage. DSC tests results can deliver, in a quick and reliable manner, estimation of SMA intrinsic properties, for example the difference in specific entropy of the austenitic and martensitic phase $\Delta s^{*}$ and the coefficient of energy of coherence $\bar{u}_{0}$, which is responsible for the splitting of $A_{s}^{0}$ and $M_{s}^{0}$ temperatures. It is energy of coherence that is responsible for the thermostatic internal dissipation of the material. This can be done using only the characteristic temperatures $A_{s}^{0}$ and $M_{s}^{0}$ and the sensible heat of PT $\Delta u^{*}$, all of which can be determined by a single DSC test. The above mentioned advantages suggest that it should be standard practice 
to determine and report the sensible heat $\Delta u^{*}$ when a DSC test is carried out.

\section{Acknowledgments}

The support of the Polish Ministry of Science and Higher Education under project No. N 501224537 "Construction of a unified thermodynamic model of one-way memory effect of large changes of shape based on experimental evidence obtained for thermomechanical loads embracing complex stress states" is sincerely acknowledged. The author thanks Professor Jan Van Humbeeck from KULeuven, Belgium, for making available the use of the technical facilities of the MTM Department of KULeuven.

\section{References}

[1] K. Otsuka, X. Ren, Intermetallics 511, 7 (1999).

[2] M.C. Carroll, Ch. Somsen, G. Eggeler, Scr. Mater. 187, 50 (2004).

[3] D. Treppmann, E. Hornbogen, J. Phys. IV 7, C5 (1997).

[4] M. Kubenová, J. Zalesak, J. Cermak, T. Bartak, A. Dlouhy, Metal, (2011), http://www.metal2012.com/files/proceedings / metal_11/lists/papers/1031.pdf .

[5] A. Biscarini, G. Mazzolai, A. Tuissi, Recent Patents Biomed. Eng. 1, 180 (2008).

[6] J. Van Humbeeck, Mater. Sci. Eng. A 273, 134 (1999).

[7] B. Raniecki, C. Lexcellent, Eur. J. Mech. A/Solids 17, 185 (1998).

[8] P.C. Su, S.K. Wu, Acta Mater. 52, 1117 (2004).

[9] G.W.H. Hohne, W. Hemminger, H.J. Flammersheim, Differential Scanning Calorimetry: An Introduction for Practitioners, 2nd ed., Springer-Verlag, Berlin 2003. 